\title{
Pancreatoblastoma: a histochemical and immunohistochemical analysis
}

\author{
Chen Hua, Xu Ke Shu, Chen Lei
}

\begin{abstract}
A case of pancreatoblastoma arising in a five year old girl was analysed using histochemical and immunohistochemical methods. The tumour was composed of tubular gland-like structures, squamoid components and some small round cells surrounding tubular structures. The cytoplasm of the small round cells and a few of the squamoid cells was positive on staining with Grimelius argyrophil stain. Immunohistochemically, tumour tissue was positive for neurone specific enolase. The cytoplasm of some of the small round cells was positive for insulin, glucagon, somatostatin, pancreatin polypeptide, thyroid stimulating hormone, follicle stimulating hormone, and neurotensin. These results suggest that this tumour arose from primitive multipotential stem cells, showing exocrine and neuroendocrine differentiation.

(F Clin Pathol 1996;49:952-954)
\end{abstract}

Keywords: pancreatoblastoma, pancreatic neoplasm, immunohistochemistry, neuroendocrine cells.

Pancreatoblastoma or infantile carcinoma of the pancreas is a very rare primary pancreatic malignancy in infants and children. In 1971, Frable et $a l^{1}$ reported a case of infantile carcinoma of the pancreas. Horie $e t a l^{2}$ in an attempt to account for the histological features of this tumour, suggested the term pancreatoblastoma. This tumour has been studied extensively using immunohistochemical techniques and electron microscopy. ${ }^{3}$ Several authors have suggested that pancreatoblastomas contain islet cell components. Here, we report a case of pancreatoblastoma in a five year old girl and discuss the possible histogenesis of this unusual neoplasm.

Department of Pathology, Qingdao Municipal Hospital Chen Hua

$\mathrm{Xu}$ Ke Shu

Department of Physiology, Qingdao Medical College Chen Lei

Correspondence to: Dr Chen Hua, Department of Pathology, Qingdao Municipal Hospital, Qingdao, 266011, Shan Dong, China. On admission, her temperature was $36.5^{\circ} \mathrm{C}$, respiratory rate was 28 , pulse 112 , and blood pressure 110/70. Physical examination revealed an $8 \times 6 \mathrm{~cm}$, fixed, firm and tender mass in the left upper abdomen. The edge of the liver was palpable $1.5 \mathrm{~cm}$ below the right costal

\section{Case report}

The patient, a five year old girl, was admitted to Qingdao Municipal Hospital in 1987 complaining of abdominal pain. The patient had had a poor appetite for the previous three months. She had diarrhoea and vomited three times during the three days prior to admission. Her past medical history was uninformative.
Table 1 Results of initial laboratory tests

\begin{tabular}{lc}
\hline Test & Value \\
\hline Haemoglobin $(\mathrm{g} / \mathrm{l})$ & 110 \\
Blood urea nitrogen $(\mathrm{g} / \mathrm{l})$ & 0.146 \\
Aspartate aminotransferase (IU/l) & 50 \\
Amylase (IU/l) & 194 \\
Serum sodium (mmol/) & 146 \\
Serum chloride (mmol/l) & 96 \\
Serum potassium (mmol/l) & 3.74 \\
$\mathrm{CO}_{2}(\mathrm{mmol} / \mathrm{l})$ & 30.2 \\
Glucose $(\mathrm{g} / \mathrm{l})$ & 0.91 \\
Alkaline phosphatase $(\mathrm{IU} / \mathrm{l})$ & 125 \\
\hline
\end{tabular}

margin. The spleen was not palpable. The patient's lymph nodes were not swollen.

Table 1 shows the results of the initial laboratory tests. Catecholamine qualitative analysis was negative. A B pattern ultrasonogram showed a $9.8 \times 5.7 \times 5.2 \mathrm{~cm}$ mass with lobules connected to the tail of the pancreas.

The mass and the surrounding pancreatic tissue were excised. The tumour had been located at the tail of the pancreas and had been connected to the transverse colon.

\section{Methods}

Tumour tissue was fixed in $10 \%$ formalin and embedded in paraffin wax. Paraffin wax sections were stained with haematoxylin and eosin, periodic acid-Schiff (PAS) with and without prior digestion with diastase, Masson's trichrome, Grimelius argyrophil stain, and Masson-Fontana stain. Sections were stained immunohistochemically using the APAAP method. Rabbit antisera were used to detect expression of human chromogranin A, insulin, gastrin, glucagon, somatostatin, pancreatin polypeptide, adrenocorticotropic hormone

\begin{tabular}{lll}
\hline Antibody & Dilution & Source \\
\hline Chromogranin A & 1 in 100 & Dako \\
ACTH & 1 in 25 & Dako \\
Insulin & 1 in 200 & Dako \\
Glucagon & 1 in 200 & Dako \\
Somatostatin & 1 in 200 & Dako \\
Pancreatin polypeptide & 1 in 100 & Dako \\
Gastrin & 1 in 300 & Dako \\
Luteinising hormone & 1 in 700 & Dako \\
TSH & 1 in 700 & Dako \\
FSH & 1 in 100 & Dako \\
Neurotensin & 1 in 100 & Dr Chen Jia Jun \\
Serotonin & 1 in 10 & Dako \\
CEA & 1 in 100 & Dako \\
Cytokeratin & 1 in 50 & Dako \\
S100 protein & 1 in 100 & Dako \\
Neurofilament & 1 in 50 & Dako \\
NSE & 1 in 100 & Dako \\
Calcitonin & 1 in 200 & Dako \\
Anti-rabbit IgG & 1 in 50 & Dako \\
Anti-mouse IgG & 1 in 50 & Dako \\
Rabbit PAP & 1 in 100 & Dako \\
Mouse PAP & 1 in 100 & Dako \\
\hline
\end{tabular}

Source: Dako, Carpinteria, California, USA.

\section{Table 2 Antibodies used with dilutions and sources}




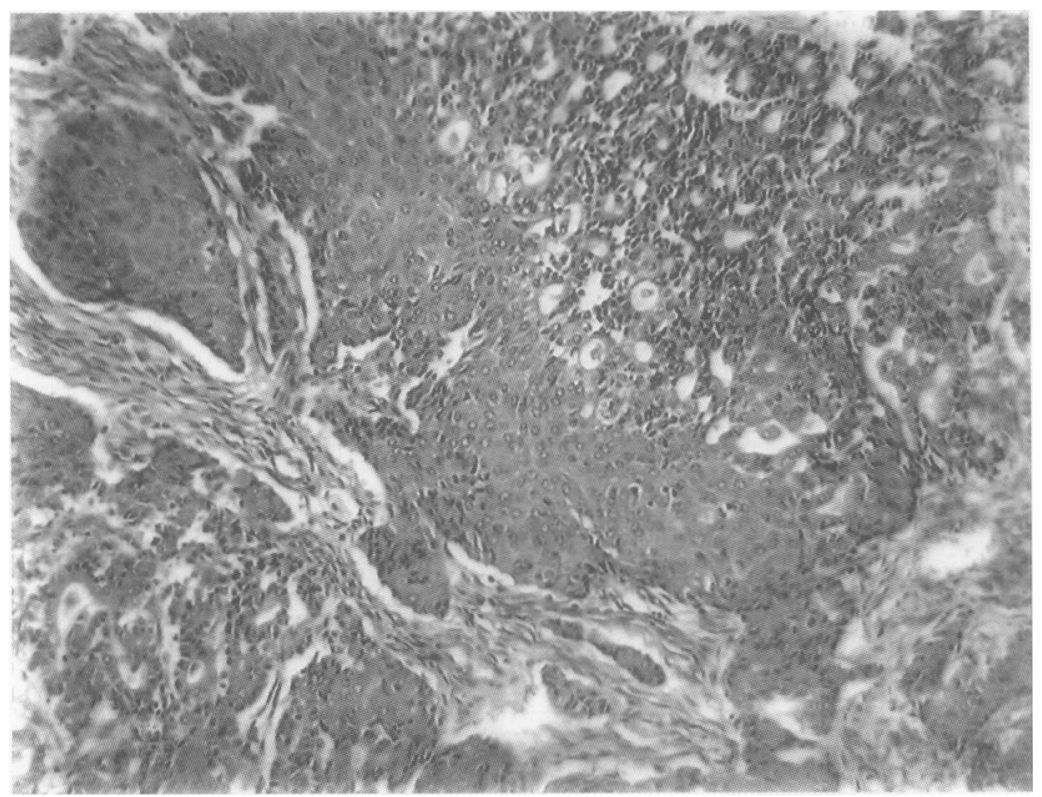

Figure 1 Section through the tumour showing tubular gland-like structures and squamoid components (haematoxylin and eosin; original magnification $\times 120$ ).

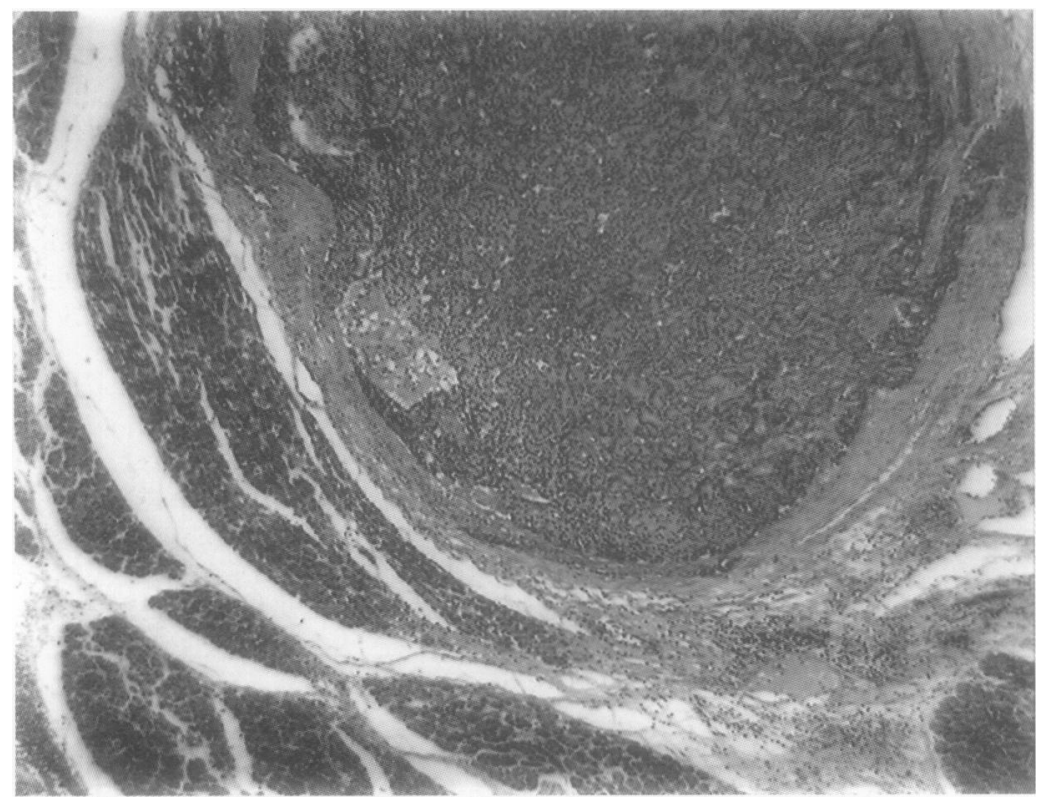

Figure 2 Tumour nodule formation with compression of surrounding pancreas (haematoxylin and eosin; original magnification $\times 120$ ).

(ACTH), thyroid stimulating hormone (TSH), neurone specific enolase (NSE), follicle stimulating hormone ( $\mathrm{FSH})$, luteinising hormone, neurotensin, and calcitonin (table 2). Mouse monoclonal antibodies were used to detect expression of serotonin (5-HT), carcinoembryonic antigen (CEA), cytokeratin, $\mathrm{S} 100$ protein, and neurofilament. Appropriate positive and negative controls were used.

\section{Pathological findings}

GROSS PATHOLOGY

The mass was a $10 \times 8 \times 6 \mathrm{~cm}$ encapsulated tumour with a smooth surface, weighing $280 \mathrm{~g}$. The cut surface revealed white, tan and yellow, medium hard and partially necrotic tissue.

LIGHT MICROSCOPY

Tumour tissue was separated by dense fibrous stroma and contained organoid lobules. Some areas were necrotic. The lobules were com- posed of tubular gland-like structures and squamoid components (fig 1). Partial glandlike structures formed lumina. The cells forming a tubular pattern had indistinct cell borders and eosinophilic cytoplasm. They had moderately round nuclei with coarse clumped chromatin and distinct borders. The squamoid cells were elongated with abundant eosi- 을 nophilic cytoplasm. Adjacent to the tubular $\overrightarrow{\vec{F}}$ structures were small round or polygonal cells, $\stackrel{0}{\rightarrow}$ with hyperchromatic nuclei and finely dispersed chromatin. There was a transition $\frac{\bar{c}}{6}$ between small round cells and gland-like cells. Mitotic figures were frequent. The tumour tis-

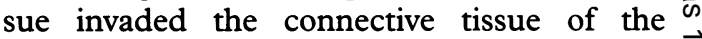
capsule and the surrounding pancreatic tissue $\overrightarrow{0}$ (fig 2). The pancreatic tissue showed increased $\overrightarrow{\vec{\omega}}$ fibrous stroma. Pancreatic acini showed extensive atrophy. PAS positive, diastase resistant $\frac{\hat{8}}{0}$ granules were seen in acinar structures. The $i$ cytoplasm of the small round cells and a few of the squamoid cells was positive on staining with Grimelius argyrophil stain.

IMMUNOHISTOCHEMICAL STAINING

The tumour tissue displayed fine granular $Z$ cytoplasmic deposition of NSE in small round $\underset{\Phi}{\widehat{T}}$ cells and in areas of the squamoid components. The cytoplasm of the small round cells was $\underset{\Phi}{\Phi}$ positive for insulin, glucagon, somatostatin, $\vec{\imath}$ pancreatin polypeptide, TSH, FSH, and neurotensin. The tubular structures were weakly positive for CEA. Staining with all of the other antibodies was negative.

\section{Discussion}

On routine light microscopy, the pancreatoblastoma seemed to be composed of organoid structures, foci of squamous components and gland-like structures. Several other investigators found the acinar structures with PAS positive granules, later confirmed to be trypsino- ? gen. Benjamin and Wright ${ }^{4}$ reported that their tumour contained $\alpha-1$-antitrypsin and speculated that this might be a marker of acinar differentiation. ${ }^{4}$

$\mathrm{Taxy}^{5}$ examined a pancreatoblastoma using 을 electron microscopy, but was unable to dem- $\frac{D}{0}$ onstrate the presence of an islet cell component. John et al detected insulin expression by ${ }^{\beta}$ immunoperoxidase and ultrastructural analy- N ses. We found that the small round cells $N$ expressed insulin, glucagon, somatostatin, pancreatin polypeptide, TSH, FSH, and neuro- 0 tensin. We confirmed that the pancreatoblas- $\frac{\mathbb{D}}{\mathbb{D}}$ toma not only had an islet cell component but $\stackrel{?}{+}$ also contained other endocrine components.

Dayal and O'Briain ${ }^{6}$ suggested that the exo- $\frac{O}{+}$ crine and endocrine pancreatic tissue in $\stackrel{\odot}{\oplus}$ pancreatoblastoma originate from tubi of pan- $\frac{\square}{2}$ creas, pancreatic exocrine components from the tubular end, and pancreatic endocrine $ᄋ$ components from the paratubi. We agree that pancreatoblastoma is an embryonal neoplasm arising from multipotential stem cells that $\stackrel{?}{?}$ mimic the embryogenesis of the pancreas with acinar and endocrine cells developing from primitive ducts. $^{?}$

Using immunohistochemistry we demonstrated the expression of TSH, FSH and 
neurotensin by tumour cells. We believe that these are primitive neoplastic endocrine components. During the differentiation process, these cells synthesise and secrete these hormones abnormally. These results provide further evidence that pancreatoblastoma is an embryonal neoplasm arising from multipotential stem cells.

We reviewed the literature and found 14 cases of pancreatoblastoma with adequate morphological description of the lesion. ${ }^{3-10}$ The ages of the patients ranged from 15 months to 13 years (average 5.5 years). More boys were affected than girls. Tumour were generally large (average $10.2 \mathrm{~cm}$ ). There was no evidence of metastasis prior to surgery in any of the patients. Two died. ${ }^{18}$ The longest follow up period was 16 years.

Our patient was followed for five years and there is no evidence of recurrence. As the tumour was encapsulated, it could be removed completely and had more favourable prognosis than pancreatic carcinoma in adults.

In conclusion, we believe that pancreatoblastoma is a unique primary pancreatic neoplasm in infants and children. This tumour, which arises from primitive multipotential stem cells, has better prognosis than pancreatic carcinomas in adults.

The authors thank Dr Chen Jia Jun, Department of Physiology, Qingdao Medical College, for providing the antisera.

1 Frable WJ, Still WJS, Kay S. Carcinoma of the pancreas, infantile type. Cancer 1971;27:667-73.

2 Horie A, Hoshinobu Y, Kotoo Y, Mirva A. Morphogenesis of pancreatoblastoma, infantile carcinoma of the pancreas. Cancer 1977;39:247-54.

3 John JB, Frederick MC, Hirikati SN. Pancreatoblastoma; A histochemical and ultrastructural analysis. Cancer 1984;53: 963-9.

4 Benjamin E, Wright DH. Adenocarcinoma of the pancreas of childhood: A report of two cases. Histopathology 1980;4: of childhood: A report of two cases. Histopathology 1980;4: 87-104.

5 Taxy JB. Adenocarcinoma of the pancreas in childhood. Cancer 1976;37:1508-18.

6 Dayal Y, O'Briain DS. The pathology of the pancreatic endocrine cells. In: Delellis RA, ed. Diagnostic immunohistochemistry. Masson Publishing, 1981:111-35.

7 Mikiro I, Toshio S, Yukihiko K, Alpha-fetoprotein-producing pancreatoblastoma: A case report. Cancer 1986;57: ducing pan

8 Cubilla AI Fitzgerald PJ. Classification of pancreatic cancer (nonendocrine). Mayo Clin Proc 1979;54:449-58.

9 Xian-Qing M. Pancreatoblastoma-infantile carcinoma of the pancreas. Chinese f Pathol 1983;1:22.

10 Jia Yu. Pancreatoblastoma: A case report. Chinese f Pathol 1987;2:143. 